\title{
Seguimiento a largo plazo del colgajo libre de peroné en la reconstrucción mandibular
}

\author{
Fibular free flap in mandible reconstruction, a long-term follow-up
}

José F. Gallegos-Hernández ${ }^{*}$, Agustín Martínez-Miramón ${ }^{1}$ y Alejandro Reyes-Vivanco²

${ }^{1}$ Departamento de Tumores de Cabeza y Cuello, Hospital de Oncología; '2Departamento de Cirugía Maxilo-facial, Hospital de Especialidades, Centro Médico Nacional siglo XXI, Instituto Mexicano del Seguro Social. Ciudad de México, México

\begin{abstract}
Resumen
Introducción: La reconstrucción mandibular con colgajo libre de peroné es el procedimiento estándar en pacientes operados de mandibulectomía por tumores óseos o de la cavidad bucal. El seguimiento a largo plazo y las complicaciones de este procedimiento son poco reportados. Objetivo: Conocer la evolución a largo plazo de pacientes con colgajo libre de peroné. Método: Evaluación clínica de pacientes con colgajo libre de peroné para reconstrucción mandibular 10 años después de la intervención. Se incluyeron operados entre 1996 y 2006. Se evaluaron clínicamente la funcionalidad oral, la integración del paciente a la dieta habitual, las complicaciones y la satisfacción estética. Resultados: Se intervinieron 87 pacientes: 70 por tumores óseos y 17 por carcinomas de la cavidad oral. Fueron exitosos 73 colgajos, y 14 tuvieron pérdida parcial o total y fueron excluidos. En todos los casos, el régimen alimentario fue similar al que tenían antes de la resección; la media para lograr la alimentación normal fue de 3 años. Ninguno requirió reoperación mayor durante el seguimiento. No hubo pérdida tardía del colgajo y la funcionalidad se consideró normal en el 100\%. El 80\% se consideraron estéticamente satisfechos. Conclusiones: El colgajo libre de peroné ofrece una alternativa segura para la reconstrucción mandibular a largo plazo.
\end{abstract}

PALABRAS CLAVE: Colgajo libre de peroné. Mandíbula. Mandibulectomía. Reconstrucción.

\begin{abstract}
Introduction: Fibula free-flap mandible reconstruction is the gold standard in mandibulectomized patients due to bone or oral cavity tumors. Long term follow-up and complications of this procedure are barely reported. Objective: To know long term outcomes of patients with fibula free-flap. Method: Clinical evaluation of patients with fibula free-flap mandible reconstruction, 10 years after procedure. Patients intervened from 1996 to 2006 were included, clinically assessing: oral functionality, habitual dietary intake, complications and aesthetic satisfaction. Results: 87 patients were included; 70 of them with bone tumors and 17 with oral carcinomas. Were successful 73 flaps, and 14 got total or partial loss, so, they were excluded. In all cases dietary regimen was similar to their original one before resection; even when in average, 3 years were necessary to get this normal intake. Any of them required major re-operation during the follow-up period and there was no late loss of the flap either. Functionality was considered normal in $100 \%$ of the cases and $80 \%$ considered themselves as aesthetically satisfied. Conclusions: Fibula free-flap offers a secure alternative for long term mandibular reconstruction.
\end{abstract}

KEY WORDS: Free fibular flap. Mandible. Mandibulectomy. Reconstruction

\author{
Correspondencia: \\ *José F. Gallegos-Hernández \\ Av. Cuauhtémoc, 330 \\ Col. Doctores \\ C.P. 06725, Ciudad de México, México \\ E-mail: gal61@ prodigy.net.mx
}

Fecha de recepción: 28-04-2018

Fecha de aceptación: 11-07-2018

DOI: $10.24875 / C I R U .18000407$
Cir Cir. 2019;87:267-271

Contents available at PubMed www.cirugiaycirujanos.com 


\section{Introducción}

El colgajo libre de peroné es el procedimiento estándar en la reconstrucción mandibular en pacientes con tumores de la cavidad oral o de origen óseo mandibular ${ }^{1,2}$. Ofrece cantidad suficiente de tejido óseo, lo que permite la reconstrucción de casi la totalidad de la mandíbula y efectuar suficiente número de osteotomías para así moldear la nueva mandíbula. Además, es un colgajo con un pedículo vascular lo bastante largo y con adecuado calibre para efectuar microanastomosis entre los vasos peroneos y los vasos receptores en el cuello ${ }^{3}$.

La arteria peronea, usualmente acompañada por dos venas peroneas, suele ser microanastomosada a la arteria facial y en ocasiones a la arteria tiroidea superior. Una anastomosis venosa suele ser suficiente para obtener adecuado drenaje venoso del colgajo, aunque cuando los vasos venosos receptores en el cuello bastan se prefiere un drenaje doble, superficial (yugular externa) e interno (afluente de yugular interna), y se realizan dos microanastomosis venosas. Tiene también la ventaja de que el colgajo puede incluir una isla de piel que permite la reconstrucción de la mucosa oral cuando es necesario ${ }^{4}$.

Es considerado como el "caballo de batalla» y el mejor método reconstructivo mandibular. El sitio donador (la pierna) prácticamente no sufre secuelas funcionales y la tasa de éxito en el sitio receptor es de alrededor del $90 \%{ }^{5}$.

Es un procedimiento no exento de complicaciones, sobre todo en el posoperatorio inmediato y mediato debido a sangrado, oclusión de las anastomosis, infección, fístula o pérdida parcial o total del colgajo, lo cual implica reintervenciones quirúrgicas y eventualmente la necesidad de otros procedimientos quirúrgicos reconstructivos ${ }^{5-7}$.

Las complicaciones a largo plazo en general se reportan escasas veces y están relacionadas principalmente con alteraciones estéticas, falta de simetría facial, mala oclusión dental, falta de rehabilitación dental, disminución de la apertura bucal o alteraciones en la nueva articulación temporomandibular en los pacientes en quienes se efectúa la resección del cóndilo mandibular en conjunto con la mandibulectomía ${ }^{7-10}$.

Las secuelas a largo plazo en el sitio donador, la extremidad inferior, son pocas. Probablemente la más importante es la dificultad para extender el primer artejo, pero esta secuela suele no suele tener impacto en la función de la extremidad; se ha reportado que la compensación de la tibia se presenta en casi todos los pacientes ${ }^{11}$.

La reconstrucción mandibular con trasplante de peroné es la mejor alternativa reconstructiva y permite al paciente reintegrarse adecuadamente a su entorno social y laboral. Incluso en defectos laterales mandibulares, la reconstrucción ósea es superior a la efectuada solo con tejidos blandos. La rehabilitación dental en el hueso trasplantado es posible y ofrece una mayor funcionalidad para la masticación y la reintegración a la dieta habitual del paciente ${ }^{12}$.

Cuando el defecto incluye la porción anterior de la mandíbula (arco anterior), el procedimiento es altamente recomendable ya que evita el colapso del mentón con la consiguiente incontinencia de la cavidad oral y el defecto estético que deteriora la calidad de vida de los pacientes.

El objetivo del presente artículo es conocer la evolución de una serie de pacientes sometidos a resección mandibular y reconstrucción con trasplante de peroné seguidos por 10 años.

\section{Método}

Efectuamos una evaluación clínica de los pacientes sometidos a mandibulectomía y reconstrucción con trasplante microvascularizado de peroné y que tenían al menos 10 años de seguimiento. Se excluyeron del análisis los pacientes que sufrieron pérdida del colgajo en el posoperatorio inmediato y tuvieron que ser reintervenidos para desmantelamiento de la reconstrucción.

Se evaluaron la tasa de éxito (porcentaje de pacientes con integración del peroné a la mandíbula sin pérdida total del implante, evaluada con ortopantomografía), la continencia o no de la cavidad oral, la capacidad para comer en público, el tipo de dieta que el paciente consumía, el tipo de rehabilitación dental logrado, las complicaciones quirúrgicas presentadas a lo largo del tiempo (como osteomielitis, secuestros óseos, necesidad de reintervenciones quirúrgicas, exposición de placas o tornillos de reconstrucción, reabsorción ósea del colgajo) y finalmente la satisfacción del propio paciente con el proceso reconstructivo.

Todos los pacientes fueron sometidos a la resección y la reconstrucción entre 1996 y 2006, y se evaluaron 10 años después. La evaluación fue clínica y se excluyó a los pacientes que no pudieron ser evaluados por cualquier motivo, por muerte o por pérdida de seguimiento. 
Tabla 1. Los defectos óseos mandibulares más frecuentes son los laterales. En cinco pacientes el defecto fue interóseo lateral y mayor de $5 \mathrm{~cm}$.

\begin{tabular}{lc}
\hline Tipo de defecto & N \\
\hline Lateral & 50 \\
Anterolateral & 10 \\
Anterior & 8 \\
Segmentectomía lateral & 5 \\
\hline
\end{tabular}

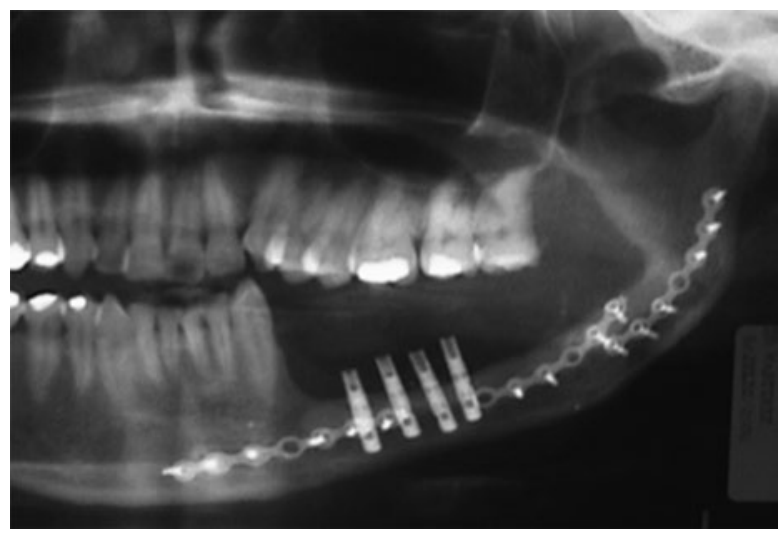

Figura 1. Los implantes osteointegrados en peroné trasplantado permiten una rehabilitación oral cercana a la normalidad.

En los pacientes sometidos a hemimandibulectomía incluyendo la desarticulación del cóndilo, este no fue sustituido por su reinserción ni prótesis; el extremo del peroné trasplantado se colocó en la cavidad condilar y esta fue cubierta con la reinserción del músculo masetero.

\section{Resultados}

Durante el periodo de 1996 a 2016 se sometieron a mandibulectomía y reconstrucción con colgajo libre de peroné 87 pacientes: 70 por tumores de origen óseo y 17 por carcinoma epidermoide de la cavidad oral.

En 73 pacientes (83\%) el trasplante fue exitoso, mientras que en 14 el colgajo tuvo que ser retirado por pérdida, necrosis o pérdida parcial. El defecto mandibular más frecuente fue el lateral. En la tabla 1 se muestran los sitios de mandibulectomía en los 73 pacientes incluidos que pudieron ser seguidos y evaluados al décimo año de su intervención.

En el momento de este reporte continúan en seguimiento 49 pacientes; 24 han fallecido durante su vigilancia, pero todos cumplieron al menos 10 años de seguimiento.
Solo 50 pacientes (68\%) tuvieron algún tipo de rehabilitación dental, la gran mayoría con placas externas y cuatro con implantes osteointegrados; todos ellos lograron una rehabilitación adecuada y no hubo pérdida de los implantes en los cuatro pacientes que los recibieron (procedimiento no efectuado en nuestra institución) (Fig. 1).

La media de tiempo en que los pacientes lograron la reintegración a un régimen alimenticio normal y similar al que tenían antes fue de 3 años. Aunque todos los pacientes toman una dieta normal y similar a la anterior al procedimiento, en el $30 \%$ de ellos la dieta fue blanda y sin la capacidad de esfuerzos importantes para la masticación.

Ninguno de los pacientes requirió durante el seguimiento retiro de todo o parte del peroné trasplantado, pero cinco tuvieron rotura de miniplacas de reconstrucción y exposición, lo que requirió su retiro. Ninguno de los pacientes presentó secuestros óseos, infecciones ni osteomielitis. Ningún paciente tuvo pérdida tardía del colgajo. En siete pacientes la apertura bucal se consideró disminuida y dos tuvieron síndrome de mala oclusión dental, pero sin repercusión en el tipo de alimentación.

Los pacientes que recibieron trasplante de peroné en la región anterior mandibular tuvieron adecuada integración ósea y en ninguno hubo incontinencia oral; el habla y la deglución fueron considerados normales.

Al interrogar a los pacientes en relación con el resultado estético, todos se consideraron satisfechos y se habían cumplido las expectativas de la reconstrucción. La función fue considerada normal, ya que ninguno requirió alimentación en forma alterna ni traqueostomía, ni hubo pérdida de la continencia oral (Figs. 2 y 3 ).

\section{Discusión}

La transferencia de tejidos autólogos microvascularizados es la mejor forma de remplazar la pérdida de tejidos óseos y blandos en los pacientes sometidos a resección de tumores del área de la cabeza y el cue$\| \mathrm{o}^{13}$. El colgajo libre de peroné, una variedad de este tipo de reconstrucción que integra tejidos blandos y hueso, es considerada la forma estándar de reconstrucción mandibular en los pacientes sometidos a mandibulectomía.

Las tasas de éxito o fracaso y las complicaciones en el posoperatorio inmediato y mediato en los pacientes que reciben un colgajo libre de peroné para 


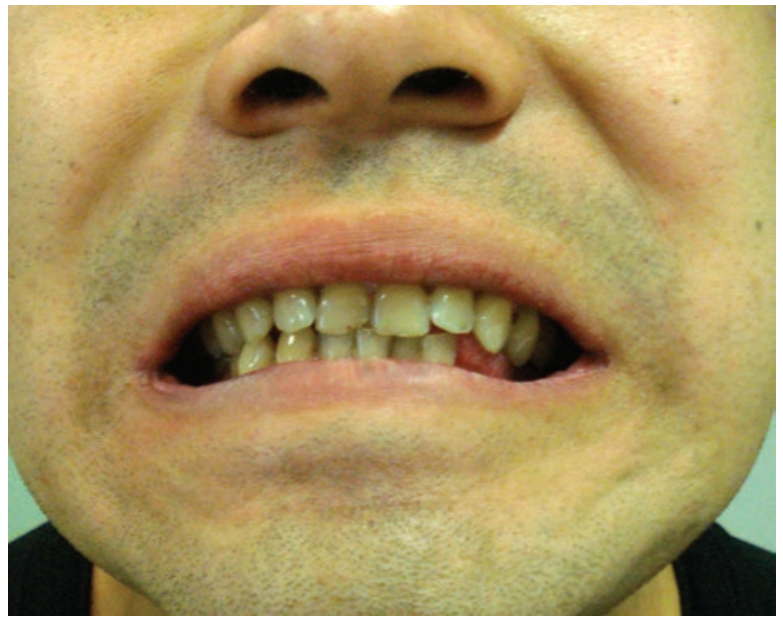

Figura 2. La oclusión es adecuada, la continencia oral es completa y el contorno facial permite una adecuada reintegración funcional y social. En estos casos con 10 años de seguimiento posoperatorio se considera un resultado estético y funcional satisfactorio, posterior a mandibulectomía y reconstrucción con colgajo de peroné.



Figura 3. Oclusión posterior a hemi-mandibulectomía izquierda, trasplante de peroné y rehabilitación dental con implantes osteo-integrados en un seguimiento mayor a 10 años.

reconstrucción mandibular son frecuentemente reportadas $^{14,15}$, pero las complicaciones a largo plazo son menos informadas en la literatura.

En la presente serie, la tasa de éxito con este colgajo es del $83 \%$. El seguimiento a largo plazo (10 años) muestra que el paso del tiempo no se asocia a complicaciones mayores, pérdida del colgajo ni pérdida de la función. La integración de los pacientes a su entorno social y laboral es adecuada incluso años después de haberse efectuado el procedimiento, lo cual confirma que este tipo de reconstrucción mandibular es una alternativa segura y funcional en los pacientes sometidos a mandibulectomía.

La zona donadora no presenta secuelas importantes ni incapacitantes a largo plazo; la disminución en el arco de la extensión del primer artejo no es incapacitante y no se traduce en morbilidad clínica ${ }^{15}$.

En los pacientes con seguimiento de más de 5 años, la complicación más importante es la fractura de las placas de reconstrucción, que requieren su retiro; sin embargo, este evento no afecta la función y suele suceder cuando el colgajo se ha integrado adecuadamente a la mandíbula residual. Si bien la rehabilitación dental con implantes osteointegrados no es un procedimiento sistemático en nuestra serie, los pacientes que la recibieron tuvieron una evolución favorable, la función masticatoria fue mejor y la oclusión dental resultó adecuada. Cuando este tipo de rehabilitación es posible, se recomienda hacerlo y puede efectuarse en el momento mismo de la realización del colgajo o cuando este ya se ha integrado a la mandíbula remanente ${ }^{16}$. En nuestra serie, la mayoría de los pacientes recibieron como rehabilitación dental la colocación de placas sobre el sitio del trasplante, lo cual no demeritó en la calidad de la función masticatoria.

En los pacientes que son sometidos a resección del cóndilo mandibular, la función de la masticación se altera (menor fuerza), pero en todos se logra la integración a la dieta habitual. No es imprescindible reinsertar el cóndilo en el peroné o utilizar una prótesis, pues la colocación del extremo del peroné en la fosa condilar y la reinserción del masetero ofrecen un resultado funcional satisfactorio; esto fue observado en la presente serie y ha sido publicado previamente por otros autores ${ }^{17}$.

Cuando se ha logrado el éxito (no complicaciones vasculares ni infecciosas en el sitio receptor) y el colgajo se ha integrado bien, formando una adecuada osteosíntesis en la mandíbula remanente, el seguimiento a largo plazo demuestra que la posibilidad de complicaciones mayores que deterioren la función es nula.

\section{Conclusiones}

Existen diversas técnicas de reconstrucción mandibular tras una mandibulectomía indicada por tumores óseos o de la cavidad oral; sin embargo, el colgajo libre de peroné parece ser el método más versátil, útil y que ofrece menor tasa de complicaciones y mejor resultado estético-funcional. Aunque no está exento de complicaciones mayores, sobre todo en el posoperatorio inmediato, y la tasa de éxito no es del $100 \%$, continúa siendo la alternativa a ofrecer especialmente en pacientes con mandibulectomía segmentaria o que incluya el arco anterior mandibular. 
Cuando se logra la integración ósea, la tasa de complicaciones a largo plazo es escasa y la evolución en el tiempo no deteriora la estética, la función ni la calidad de vida de los pacientes.

\section{Responsabilidades éticas}

Protección de personas y animales. Los autores declaran que para esta investigación no se han realizado experimentos en seres humanos ni en animales.

Confidencialidad de los datos. Los autores declaran que han seguido los protocolos de su centro de trabajo sobre la publicación de datos de pacientes.

Derecho a la privacidad y consentimiento informado. Los autores han obtenido el consentimiento informado de los pacientes y/o sujetos referidos en el artículo. Este documento obra en poder del autor de correspondencia.

\section{Conflicto de intereses}

Los autores declaran que no tienen conflicto de intereses.

\section{Bibliografía}

1. Bosc R, Hersant B, Carloni R, Niddam J, Bouhassira J, Kermadec H, et al. Mandibular reconstruction after cancer: an in-house approach to manufacturing cutting guides. Int J Oral Maxillofac Surg. 2017;46:24-31.

2. Ton Van J, Gallegos JF, Sarini J, Buisset E, Demaille A, Lefebvre JL. Impact del envahissement mandibulaire des cancers de la cavité buccale. En: Le mandibule en carcinologie.Societé Française de Carcinologie Cérvico Faciale (SFCCF) París: Masson; 1994:59-64.

3. Hidalgo DA. Fibula free flap: a new method of mandible reconstruction. Plast Reconstr Surg. 1989;84:71-9.

4. Chen YK, Zheng BG, Zhu JM, Zheng BX, Gu YD, Wu MM, et al. Microsurgical anatomy of the lateral skin flap of the leg. Ann Plast Surg. 1985;15:313-8.

5. Lee M, Chin RY, Esick GD, Sritharan N, Paramaesvaran S. Outcomes of microvascular free flap reconstruction for mandibular osteoradionecrosis: a systematic review. J Craniomaxillofac Surg. 2015;43:2026-33.

6. Gallegos-Hernández JF, Martínez-Miramón A. Trasplante de peroné para reconstrucción mandibular en pacientes sometidos a mandibulectomía por tumores óseos y de la cavidad bucal. Experiencia de 10 años. Gac Med Mex. 2008;144:85-90.

7. Lodders JN, Schulten EA, de Visscher JG, Forouzanfar T, Karagozoglu $\mathrm{KH}$. Complications and risk after mandibular reconstruction with fibular free flap in patients with oral squamous cell carcinoma: a retrospective cohort study. J Reconstruct Microsurg. 2016;32:455-63.

8. Jarefors $E$, Hansson T. Functional outcome in 17 patients whose mandibles were reconstructed with fibular free flaps. J Plast Surg Hand Surg. 2016;15:1-4.

9. Wijbenga JG, Schepers RH, Werker PM, Witjes MJ, Dijstra PU. A systematic review of functional outcome and quality of life following reconstruction of maxillofacial defects using vascularized free fibula flaps and dental rehabilitation reveals poor data quality. J Plast Reconstr Aesthet Surg. 2016;69:1024-36.

10. Taylor Gl, Corlett RJ, Ashton MW. The evolution of free vascularized bone transfer: a 40-year experience. Plast Reconstr Surg. 2016;137:1292-305.

11. van den Heuvel SC, van der Veen FJ, Winters HA. The effect of flexor hallucis longus harvest on hallux function: a retrospective cross-sectional cohort styudy. J Plast Reconstr Aesthet Surg. 2014;67:986-91.

12. Fang W, Liu YP, Ma Q, Liu BL, Zhao Y. Long-term results of mandibular reconstruction of continuity defects with fibula free flap and implant-borne dental rehabilitation. In J Oral Maxillofac Implants. 2015;30:169-78.

13. Cannady SB, Lamarre E, Wax MK. Microvascular reconstruction: evidence-based procedures. Facial Plast Surg Clin North Am. 2015;23:347-56.

14. Wu H, Liu F, Ji F, Guo M, Wang Y, Cao M. Identification of independent risk factors for complications: a retrospective analysis of 163 fibular fee flaps for mandibular reconstruction. J Oral Maxilofac Surg. 2018; 76:1571-7.

15. Houdoiri N, Feuvrier D, Pauchot J, Decavel P, Sagawa Y. Donor site morbidity after vascularized fibula free flap: gait analysis during prolonged walk conditions. In J Oral Maxillofac Surg. 2018;47:309-15.

16. Sozzi D, Novelli G, Silva R, Connely ST, Tartaglia GM. Implant rehabilitation in fibula-free flap reconstruction: a retrospective study of cases at 1-18 years following surgery. J Craniomaxillofac Surg. 2017;45:1655-61.

17. Garvvanis A, Anterriotis D, Kakagia D. Mandibular condyle reconstruction with fibula free-flap transfer: the role of maseter muscle. J Craniofac Surg. 2017;28:1955-9. 